OPEN ACCESS

Edited by:

Lei Wang,

Changsha University, China

Reviewed by:

Xinguo Lu,

Hunan University, China

Pu-Feng Du,

Tianjin University, China

${ }^{*}$ Correspondence:

Xuan Xiao

jdzxiaoxuan@163.com

Wang-Ren Qiu

qiuone@163.com

Specialty section:

This article was submitted to

Computational Genomics,

a section of the journal

Frontiers in Genetics

Received: 08 July 2021

Accepted: 02 August 2021

Published: 09 September 2021

Citation:

Zheng J, Xiao X and Qiu W-R (2021) iCDI-W2vCom: Identifying the Ion Channel-Drug Interaction in Cellular Networking Based on

word2vec and node2vec.

Front. Genet. 12:738274.

doi: 10.3389/fgene.2021.738274

\section{iCDI-W2vCom: Identifying the lon Channel-Drug Interaction in Cellular Networking Based on word2vec and node2vec}

\author{
Jie Zheng, Xuan Xiao* and Wang-Ren Qiu* \\ Department of Computer Engineering, Jingdezhen Ceramic Institute, Jingdezhen, China
}

Ion channels are the second largest drug target family. Ion channel dysfunction may lead to a number of diseases such as Alzheimer's disease, epilepsy, cephalagra, and type II diabetes. In the research work for predicting ion channel-drug, computational approaches are effective and efficient compared with the costly, labor-intensive, and time-consuming experimental methods. Most of the existing methods can only be used to deal with the ion channels of knowing 3D structures; however, the 3D structures of most ion channels are still unknown. Many predictors based on protein sequence were developed to address the challenge, while most of their results need to be improved, or predicting web servers are missing. In this paper, a sequence-based classifier, called "iCDI-W2vCom," was developed to identify the interactions between ion channels and drugs. In the predictor, the drug compound was formulated by SMILES-word2vec, FP2-word2vec, SMILES-node2vec, and ECFPs via a 1184D vector, ion channel was represented by the word2vec via a 64D vector, and the prediction engine was operated by the LightGBM classifier. The accuracy and AUC achieved by iCDI-W2vCom via the fivefold cross validation were $91.95 \%$ and 0.9703 , which outperformed other existing predictors in this area. A user-friendly web server for iCDI-W2vCom was established at http://www.jci-bioinfo.cn/icdiw2v. The proposed method may also be a potential method for predicting target-drug interaction.

Keywords: ion channels, word2vec, node2vec, data augmentation, LightGBM

\section{INTRODUCTION}

Ion channels are pore-forming membrane proteins that mediate the transport of ions in all living cells (Green, 1999) by controlling cell signaling during the change of the cellular physiology in organs (Gabashvili et al., 2007). For example, ion channels regulate the membrane potential by mediating the permeation of specific ion species through their transmembrane pores (Sumino et al., 2019). On other hand, dysfunction of ion channels may lead to over 55 different channelopathies (Qiang et al., 2018), such as epilepsy, arrhythmia, and type II diabetes (Tinaquero et al., 2020). It is also believed that the majority of patients with thyroid diseases and cardiac arrhythmia are ion channel blockers (Roepke et al., 2009) such that ion channels become important therapeutic targets.

As an essential step of drug discovery procedure, the identification of ion channel-drug interaction has lately become a hot topic issue since it involves costly, time-consuming, and 
challenging work during the development of new medicine (Knowles and Gromo, 2003). It has been reported that ionotropic glutamate receptor subfamily core is formed by two transmembrane helices and an intracellular reentrant pore helix (Amin et al., 2018); voltage-gated ion channels, including potassium channels and calcium channels, consist of six transmembrane helices (Kaufmann et al., 2019). Therefore, ion channels may be analyzed by using conventional methods of protein, and identification of ion channel-drug interactions inherently is a protein-drug interaction problem. There are many unpaired small molecule compounds for finding potentially new medications; many state-of-the-art computational methods have been developed to discover new drugs in the past few years (Xiao et al., 2013; Chen et al., 2018; Wang et al., 2020). Yamanishi et al. (2008) used statistical approach to predict the interaction between drugs and four targets on the base of the similarity. Wang et al. (2020) proposed a sequence-based method for identifying the protein-drug interaction. Since ion channel-drug interaction involves two substances, the methods that combine the biological information of ion channels and the chemical information of drugs are often used, and proper representation of proteins and drugs is essential to identify ion channel-drug with high efficiency.

For the feature extraction from protein, there are many classic algorithms applied to extract the feature from amino acid sequence, such as one hot encoding (Wang et al., 2019), composition of k-spaced amino acid pairs (CKSAAP; Chen et al., 2006), amino acid composition (AAC; Reczko and Bohr, 1994), and pseudo amino acid composition (PseAAC; Chou, 2005). The technology of natural language processing (NLP) was used to deal with secondary-structure prediction and subcellular localization (Elnaggar et al., 2020) in proteomics area. Furthermore, the deep learning techniques have been used to extract sequence features for protein-drug interaction. In order to get a protein feature (Wang et al., 2020), protein sequences were encoded using one hot encoding, and the information is then fed into a deep learning model, such as recurrent neural network (RNN), long short-term memory (LSTM), and gated recurrent unit (GRU; Shen et al., 2020).

For the processing of drug molecules, a variety of descriptors are used to represent drugs to fill the gap in analyzing the $3 \mathrm{D}$ structure for drugs, such as two-dimensional molecule graph (Bemis and Kuntz, 1992), MOL file (Qiu et al., 2020), simplified molecular-input line-entry system (SMILES; Jaeger et al., 2017), fingerprint and global descriptions of molecular by biophysical and chemical properties including the molecular weight (MW) and the partition coefficient for lipophilicity (Clark et al., 2019; Daina and Zoete, 2019). In these descriptors, drug molecules are usually represented with SMILES or Morgan fingerprint (Morgan FPs). The representation of SMILES string involves four overall steps: graph mol structure normalization, canonical labeling, tree traversal, and SMILES generation (O'Boyle, 2012), which is usually the start step for many computational methods such as recurrent neural networks (RNNs; Karimi et al., 2019), convolutional neural networks (CNNs; Huang et al., 2020), and graph neural networks (GNN; Tsubaki et al., 2019). Take extended connectivity fingerprints (ECFPs) as an example for
Morgan FPs; all substructures around all heavy atoms of a molecule within a defined radius are generated and assigned to a unique identifier (called Morgan identifier), which would be compressed into a shorter fixed-length string (Zhou et al., 2020). The drug's MOL file or SMILES can be acquired from http: //www.kegg.jp/kegg/ or https://www.ebi.ac.uk/chembl/, and the software called OpenBabel ${ }^{1}$ could be used to convert the MOL file or SMILES into molecular fingerprint files in multiple formats: FP2, FP3, FP4, and MACSS.

Some novel encoding techniques were provided for proteins and drugs based on word2vec algorithm. As word2vec could map a class $\mathrm{X}$ of objects into a latent vector space where the geometric relationship is characterized by the semantic relationship between the objects (Grohe, 2020), it has been adapted to classify the protein sequences of protein families and predict the localization of proteins and the compound properties of drugs (Jaeger et al., 2017; Yang et al., 2018). Jaeger et al. (2018) proposed that the word2vec may identify the interaction between drugs and target proteins based on the amino acid sequences of proteins and the Morgan fingerprints of drugs. Zhang et al. (2019) further proposed a new predictor by using the amino acid sequences of proteins and the SMILES strings of drugs.

The study of ion channel-drug interaction networks is an important topic for drug development, while the computational prediction accuracies cannot meet the practical needs. Although deep learning methods are widely used in protein-target prediction, it is still in the exploratory stage for identifying ion channel-drug interaction. In addition, many research focused

\footnotetext{
${ }^{1}$ http://openbabel.org/
}

TABLE 1 | Five physicochemical property codes for each of the 20 native amino acids.

\begin{tabular}{lrrrrr}
\hline Amino acid & \multicolumn{5}{c}{ Five physicochemical property codes } \\
\cline { 2 - 6 } & AAindex1 & AAindex2 & AAindex3 & AAindex4 & AAindex5 \\
\hline A & 1.8 & 89.09 & 6.00 & 9.69 & 2.34 \\
C & 2.5 & 121.15 & 5.05 & 8.35 & 1.92 \\
D & -3.5 & 133.10 & 2.77 & 9.60 & 1.88 \\
E & -3.5 & 147.13 & 3.22 & 9.67 & 2.10 \\
F & 2.8 & 165.19 & 5.48 & 9.18 & 2.16 \\
G & -0.4 & 75.07 & 5.97 & 9.78 & 2.35 \\
H & -3.2 & 155.16 & 7.59 & 9.17 & 1.82 \\
I & 4.5 & 131.17 & 6.02 & 9.68 & 2.36 \\
K & -3.9 & 146.19 & 9.74 & 9.18 & 2.16 \\
L & 3.8 & 131.17 & 5.98 & 9.60 & 2.36 \\
M & 1.9 & 149.21 & 5.74 & 9.21 & 2.28 \\
N & -3.5 & 132.12 & 5.41 & 8.80 & 2.02 \\
P & -1.6 & 115.13 & 6.30 & 10.64 & 1.95 \\
Q & -3.5 & 146.15 & 5.65 & 9.13 & 2.17 \\
R & -4.5 & 174.20 & 10.76 & 8.99 & 1.82 \\
S & -0.8 & 105.09 & 5.68 & 9.21 & 2.19 \\
T & -0.7 & 119.12 & 5.66 & 9.10 & 2.09 \\
V & 4.2 & 117.15 & 5.96 & 9.62 & 2.32 \\
W & -0.9 & 204.24 & 5.89 & 9.44 & 2.43 \\
Y & -1.3 & 181.19 & 5.66 & 9.11 & 2.20 \\
& & & & &
\end{tabular}


on constructing a complex neural network to extract interaction information, but for a method to encode a sequence, which is a crucial point of protein and drug representation, it gets rare attention. Thus, this paper was initiated in an attempt to develop a new powerful predictor based on the sequences of ion channels and the SMILES of drugs. There are four innovative characteristics of this work: (1) To get a better representation of protein, amino acid sequences were divided into words (k-grams) and encoded with the AAindex, which would be fed into word2vec to get distributed representations vectors of words. (2) To find the best way for the representation, two major descriptions, SMILES (SMILES_word2vec), and FP2 (FP2_word2vec), were separately tested for comparison on the basis of several combined features. (3) To augment the training dataset and get more information about the linking between different functional groups, the $\mathrm{RDKit}^{2}$, an

${ }^{2}$ http://www.rdkit.org/ open source chemistry informatics and machine learning toolkit, was used to generate different SMILES strings for the same molecule, and finally note $2 \mathrm{vec}$ was applied to generate drug vectors (SMILES_node2vec). (4) To make full use of the drug and protein features mentioned above, the feature combination was performed deeply, and the prediction results improved significantly.

\section{MATERIALS AND METHODS}

\section{Benchmark Dataset}

As more and more interactive pieces of information are in the database, such as DrugBank, KEGG, STITCH, ChEMBL, and TTD, many deep research studies have been carried out in drug discovery. In this work, the identification of ion channel-drug interaction is defined as a supervised prediction task in which a pair of counterparts interact with each other in the drug-target

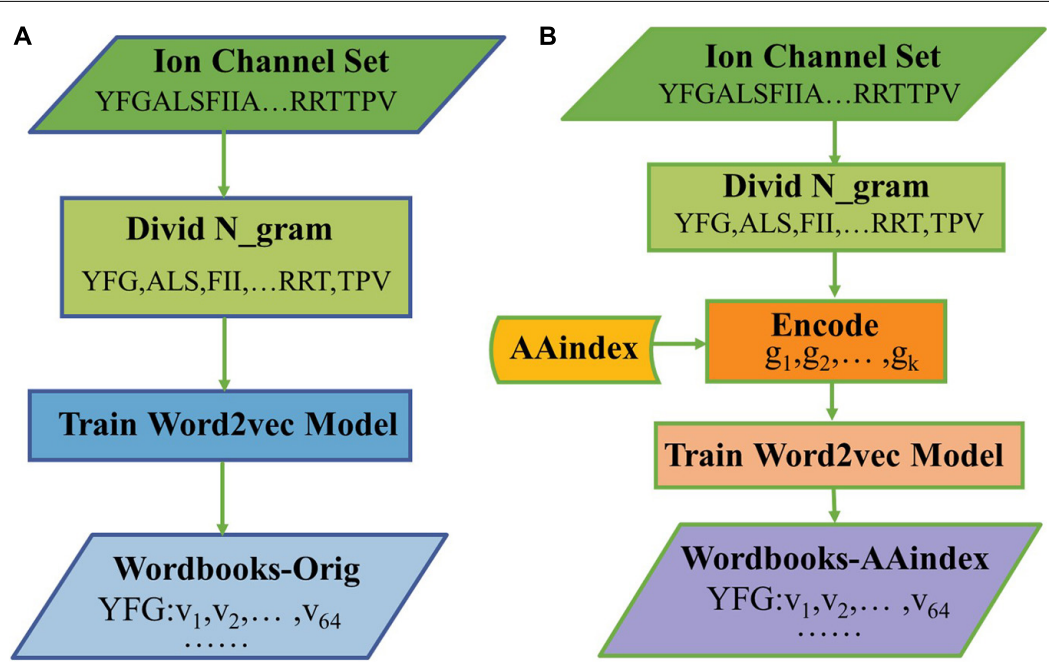

FIGURE 1 | The processes of constructed (A) Wordbooks-Orig and (B) Wordbooks-AAindex.

A
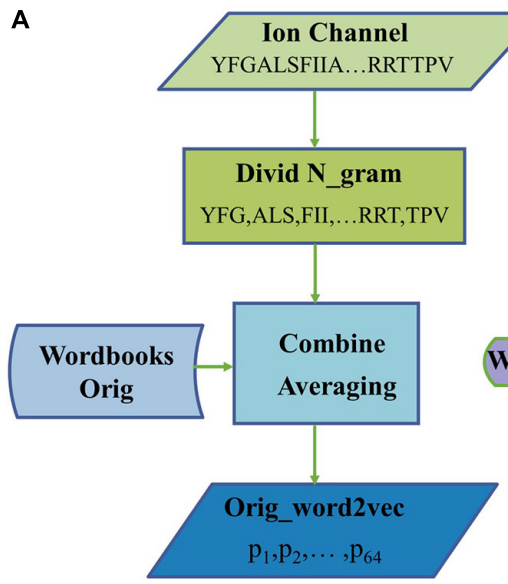

B

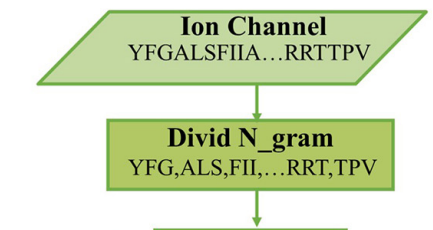

AAindex

Encod

$\mathrm{g}_{1}, \mathrm{~g}_{2}, \ldots, \mathrm{g}_{\mathrm{k}}$

Wordbooks-AAindex
Combine

Averaging

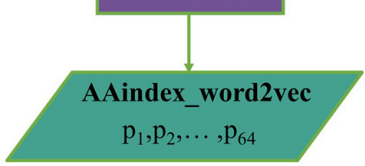

FIGURE 2 | The processes of generating protein representation through (A) Orig_word2vec and (B) AAindex_word2vec. 
networks. The established KEGG database is utilized to define the pair of counterparts as it has an amount of interaction information of drugs and drug targets.

In this work, the benchmark dataset $S$ is defined by:

$$
S=S^{+} \cup S^{-}
$$

where $\mathrm{S}^{+}$is the set of interactive ion channel-drug pairs, and $\mathrm{S}^{-}$is the set of non-interactive ion channel-drug pairs, and the symbol $\cup$ represents the union in the set theory. The positive subset $\mathrm{S}^{+}$contains 1,476 ion channel-drug pairs collected by Yamanishi et al. (2008).
To build the negative dataset, the approach was performed with the following steps: (i) Each pair in subset $\mathrm{S}^{+}$was separated (drug ID and ion channel ID) into a single ion channel and drug. (ii) Each of the single ion channels was re-coupled with each of the single drug; therefore, the drug and ion channel are put into synthesized pairs in such a way. Those pairs that were in $\mathrm{S}^{+}$were removed, and it was made sure that none of the pairs that were in $\mathrm{S}^{+}$appeared in $\mathrm{S}^{-}$. (iii) The synthesized pairs were randomly picked until the number of selected pairs was the same as the number of pairs in $\mathrm{S}^{+}$. The dataset $\mathrm{S}^{-}$contains 1,476 non-interactive ion channel-drug pairs.

An independent validation test is applied to evaluate the developed predictor for avoiding the overfitting of data from
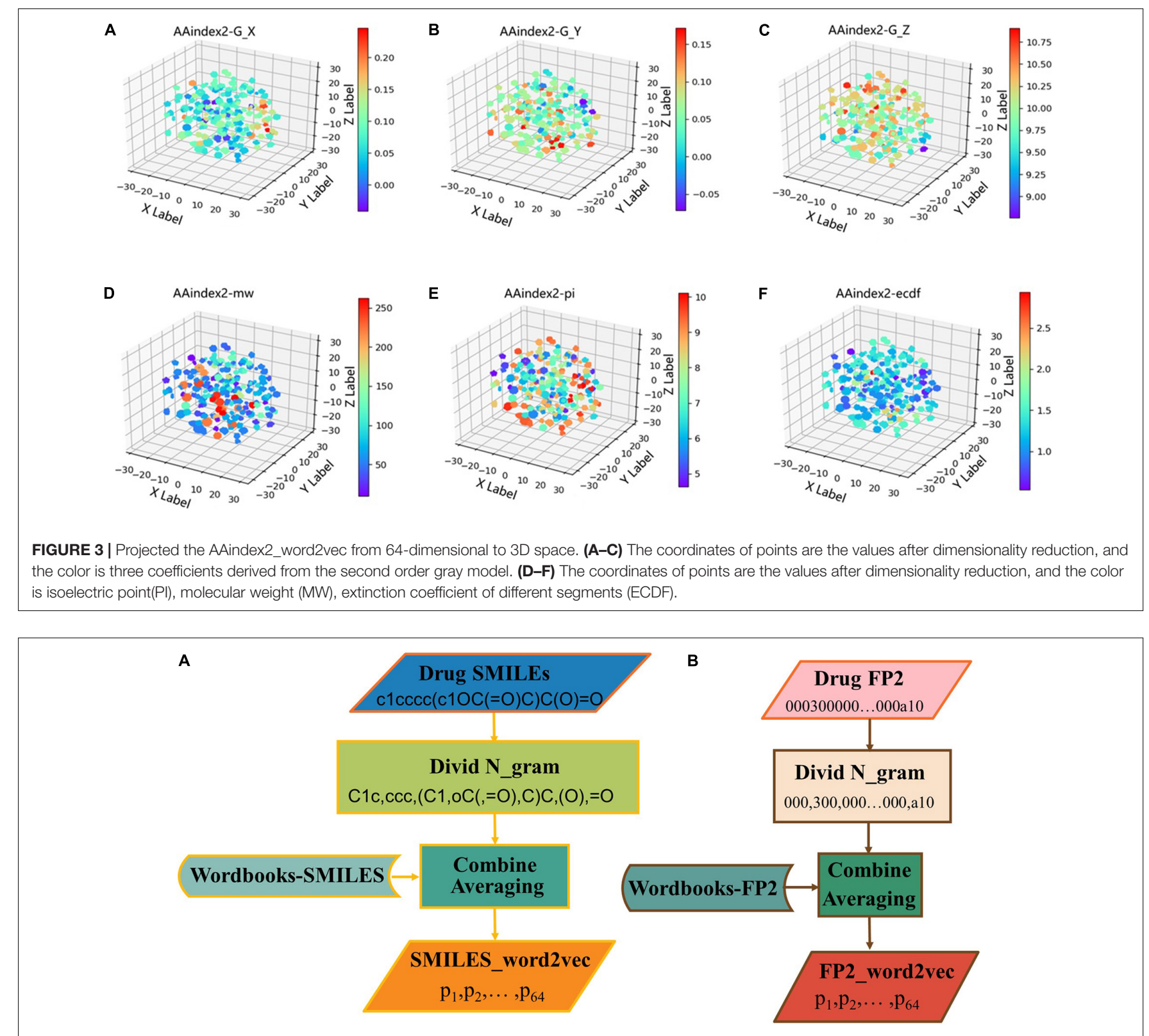

B

FIGURE 4 | The processes of generating (A) SMILES_word2vec and (B) FP2_word2vec. 


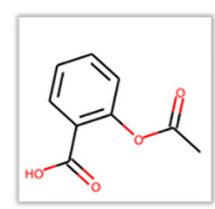

$\operatorname{c1cccc}(\operatorname{coc} 0 \mathrm{C}(=0) \mathrm{C}) \mathrm{C}(0)=\mathrm{O}$

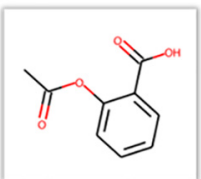

$\operatorname{c1cccc}(\operatorname{c1C}(=0) 0) O C(C)=0$

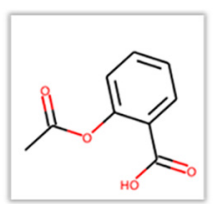

$c 1(O C(C)=0) c(\operatorname{cccc} 1) C(0)=0$

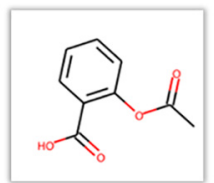

$\operatorname{c1cc}(c(c c 1) C(=0) 0) 0 C(C)=0$
FIGURE 5 | Generation of different SMILES strings for the same molecule.

the reference (Yamanishi et al., 2008). The validation dataset, denoted as Check808, contains 404 interactive pairs and 404 non-interactive pairs. These pairs consist of the ion channels in $S$ and new drug targets taken from the KEGG database. Any pairs have to be removed from the validation dataset if they appeared in the benchmark dataset.

Nuclear receptors (NRs) are another frequent target for drug development, but drug-NR pairs are more difficult in the protein-drug predict task. The dataset of NRs is used to verify the feature extraction method and the robustness of iCDI-W2vCom. The NR dataset contains a positive subset of 86 interactive drugNR pairs, taken from the reference (Yamanishi et al., 2008) and a negative subset of 86 non-interactive pairs. The non-interactive pairs are different from the interactive pairs.

\section{Measurement}

In the experiment, the performances of the predictor were evaluated with the following four metrics: accuracy (Acc), sensitivity (Sn), precision (Prec), and Matthews correlation coefficient (MCC; Jiao and Du, 2016). They were applied to evaluate the models and are shown in formula (2).

$$
\left\{\begin{array}{l}
A c c=\frac{T P+T N}{T P+T N+F P+F N} \\
\text { Sen }=\frac{T P}{T P+F N} \\
\text { Prec }=\frac{T P}{T P+F P} \\
M C C=\frac{T P \times T N-F P \times F N}{\sqrt{(T P+F P)(T P+F N)+(T N+F P)(T N+F N)}}
\end{array}\right.
$$

\section{Representation of Ion Channel}

The ion channel with the sequence length $l$ is formulated in the following format:

$$
G=R_{1} R_{2} R_{3} R_{4} R_{5} R_{6} \ldots R_{3 \mathrm{j}+1} R_{3 \mathrm{j}+2} R_{3 \mathrm{j}+3} \ldots R_{1}
$$

where $R_{1}$ represents the first residue in ion channel sequence, $R_{2}$ represents the second, ..., and $R_{l}$ represents the $l$-th one. How can we extract sequence information to represent an ion channel? We should translate a protein sequence into a digital vector that can well represent an ion channel.

In the article, three amino acids are divided into one word to construct the wordbook. As shown in the following example, a sequence of nine amino acids can be divided into three sets of non-overlapping 3-gram. Then $G$ would be grouped as:

$$
G=\left(R_{1} R_{2} R_{3}\right)\left(R_{4} R_{5} R_{6}\right) \ldots\left(R_{3 \mathrm{j}+1} R_{3 \mathrm{j}+2} R_{3 \mathrm{j}+3}\right)
$$

where $G=G_{1} G_{2} \ldots G_{j} \ldots G_{L} G_{L+1}, L=[l / 3], L$ is a round down of $l / 3$, and $G_{L+1}$ may be $\varnothing$ or only contains one or two residues, which are due to the remainder of $l / 3$.
A

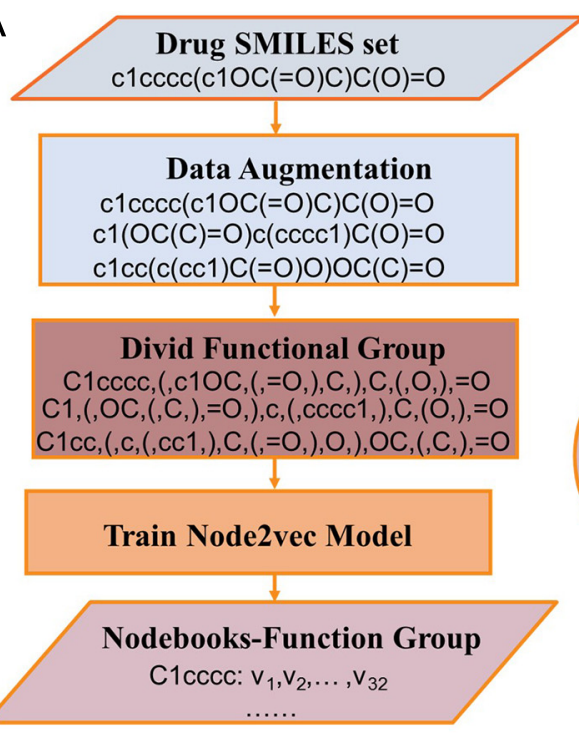

B

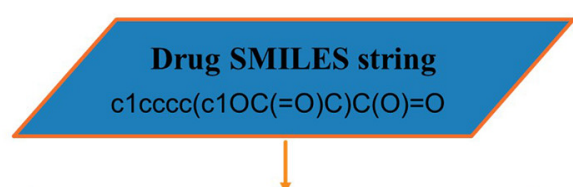

Divid Functional Group

C1cccc, $(, \mathrm{c} 1 \mathrm{OC},(,=\mathrm{O}),, \mathrm{C}),, \mathrm{C},(, \mathrm{O}),,=\mathrm{O}$

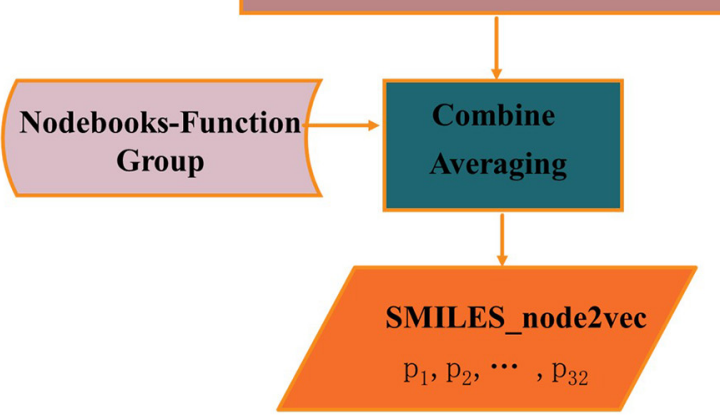

FIGURE 6 | The processes of (A) constructing Wordbooks-Orig and (B) generating SMILES_node2vec. 
TABLE 2 | Profile of the six learners.

\begin{tabular}{|c|c|c|c|c|c|c|}
\hline & AAindex1_word2vec & SMILES-word2vec & FP2-word2vec & SMILES-node2vec & ECFP & Number of features \\
\hline Learn-1 & $\sqrt{ }$ & $\sqrt{ }$ & & & & 128 \\
\hline Learn-2 & $\sqrt{ }$ & & $\sqrt{ }$ & & & 128 \\
\hline Learn-3 & $\sqrt{ }$ & $\sqrt{ }$ & & $\sqrt{ }$ & & 160 \\
\hline Learn-4 & $\sqrt{ }$ & & $\sqrt{ }$ & $\sqrt{ }$ & & 160 \\
\hline Learn-5 & $\sqrt{ }$ & $\sqrt{ }$ & $\sqrt{ }$ & $\sqrt{ }$ & & 224 \\
\hline Learn-6 & $\sqrt{ }$ & $\sqrt{ }$ & $\sqrt{ }$ & $\sqrt{ }$ & $\sqrt{ }$ & 1,248 \\
\hline
\end{tabular}

ECFP, extended-connectivity fingerprints.

The AAindex database indexes are the biophysical and chemical properties of amino acids and pairs of amino acids ${ }^{3}$ (Zhou et al., 2020). In this paper, five groups of AAindex were selected for the experiment, which are the same as the reference (Wang et al., 2020), and the corresponding values of amino acids are shown in Table 1. The AAindex1 physicochemical property stands for "hydropathy index," AAindex2 for "molecular weight," AAindex 3 for "isoelectric point (PI)," AAindex4 for "pK-N," and AAindex 5 for "pK-C."

With the AAindex values, the ion channel sequence $G$ would be encoded into a vector shown as follows:

$$
G=\left(g_{1} g_{2} \ldots g_{\mathrm{j}} \ldots g_{\mathrm{L}} g_{\mathrm{L}+1}\right), g_{\mathrm{j}}=\frac{\sum_{\mathrm{R} \in \mathrm{G}_{\mathrm{j}}} \rho(R)}{\left\|G_{\mathrm{j}}\right\|}
$$

where $\rho(R)$ is the AAindex value of reside $R$, and $\left\|G_{j}\right\|$ is the number of residues in group $G_{j}, j=1,2, \ldots, L(L+1$ when the

${ }^{3}$ http://www.genome.jp/aaindex/

TABLE 3 | Performance of different protein representations on the ion channel dataset.

\begin{tabular}{lccccc}
\hline Protein feature & AUC & Acc (\%) & Prec (\%) & Sen (\%) & MCC \\
\hline Orig_word2vec & 0.9720 & 91.29 & 90.75 & 91.91 & 0.8262 \\
AAindex1_word2vec & 0.9703 & 91.95 & $\mathbf{9 1 . 1 8}$ & 92.95 & 0.8402 \\
AAindex2_word2vec & 0.9717 & 91.69 & 90.76 & 92.72 & 0.8344 \\
AAindex3_word2vec & 0.9700 & 91.77 & 91.15 & 92.41 & 0.8355 \\
AAindex4_word2vec & 0.9676 & 91.22 & 90.85 & 91.65 & 0.8247 \\
AAindex5_word2vec & 0.9682 & 91.22 & 90.32 & 92.20 & 0.8248 \\
All_word2vec & $\mathbf{0 . 9 7 3 0}$ & $\mathbf{9 2 . 1 3}$ & 91.17 & $\mathbf{9 3 . 1 3}$ & $\mathbf{0 . 8 4 3 0}$ \\
\hline $\begin{array}{l}\text { ACC, accuracy; Prec, precision; Sen, sensitivity; } \\
\text { correlation coefficient. Bold values mean that they }\end{array}$ are the best scores \\
compared with other methods.
\end{tabular}

TABLE 4 | Performances of different drug descriptions on the ion channel dataset.

\begin{tabular}{lccccc}
\hline & AUC & Acc (\%) & Prec (\%) & Sen (\%) & MCC \\
\hline Learner-1 & 0.9598 & 90.24 & 88.98 & 91.61 & 0.8054 \\
Learner-2 & 0.9675 & 91.26 & 90.26 & 92.33 & 0.8256 \\
Learner-3 & 0.9625 & 91.33 & 90.41 & 92.36 & 0.8271 \\
Learner-4 & 0.9703 & 91.25 & 90.16 & 92.46 & 0.8257 \\
Learner-5 & 0.9696 & 91.51 & 90.16 & $\mathbf{9 3 . 0 3}$ & 0.8310 \\
Learner-6 & $\mathbf{0 . 9 7 0 3}$ & $\mathbf{9 1 . 9 5}$ & $\mathbf{9 1 . 1 8}$ & 92.95 & $\mathbf{0 . 8 4 0 2}$ \\
\hline
\end{tabular}

Bold values mean that they are the best scores compared with other methods. remainder of $l / 3$ is not equal to zero). Therefore, a new corpus of words is constructed through AAindex indices. The corpus may reduce the number of words made of amino acids string. For example, a word made of AAindex indices may take the place of a triplet composed of amino acids $\mathrm{D}, \mathrm{E}$, and $\mathrm{F}$ with a total of nine words. Such an expression may also combine triplicates with similar properties together. In particular, the hydrophilic coefficient is used to code the triplicates; the words "EFG" and "DFG" will be combined into the same word.

Although word2vec is an unsupervised method, here, an auxiliary prediction task was defined to train the word representation model with one of the following two approaches: (1) continuous bag-of-words (CBOW), which may predict a word from the context words, and (2) Skip-gram, which predicted the context based on a word. In CBOW, the order of words in the context is not important due to the bag-of-words assumption, while the adjacent words are assigned with higher weights in Skip-gram. We mainly used the Skip-gram model to train the word2vec model.

The classical Skip-gram model consists of an input layer, projection layer, and output layer. The model learns information from corpus and stores the derived knowledge in weights $\theta$. The positive samples of Skip-gram model are words $g_{I}$ and their contexts $C\left(g_{I}\right)$. Contexts of a word $g_{I}$, which was derived from a window of size $\mathrm{k}$ around the word: $C(g)=g_{i-k}, \ldots, g_{i-1}, g_{i+1}, \ldots, g_{i+k}$, where the window size $\mathrm{k}$ is a parameter for word2vec; the negative samples are generated by relatively simple method called negative sampling.

The hyperparameters of Skip-gram were set as follows: the embedding dimension is $d=64$, the context window size is $k=4$, and the number of negative examples is $k=8$. After training for 30 epochs, we get a final wordbook. The process of constructing the original triplicate workbooks (WordbooksOrig) is shown in Figure 1A, as a result, each word would be represented with a 64-dimensional vector, and each word and its corresponding vector are storied in "Wordbooks-Orig." The process of constructing a Wordbooks-AAindex is shown in Figure 1B. Finally, each word encoded with AAindex indices is represented with a 64-dimensional vector; the words and their vectors are storied in "Wordbooks-AAindex."

Figure 2 illustrates the process of generating protein representation through Orig_word2vec and AAindex_word2vec, respectively. In Figure 2A, amino acid sequences were divided into 3-gram, and then looked up the "Wordbooks-Orig" to obtain a vector for every word. The representation for a protein 


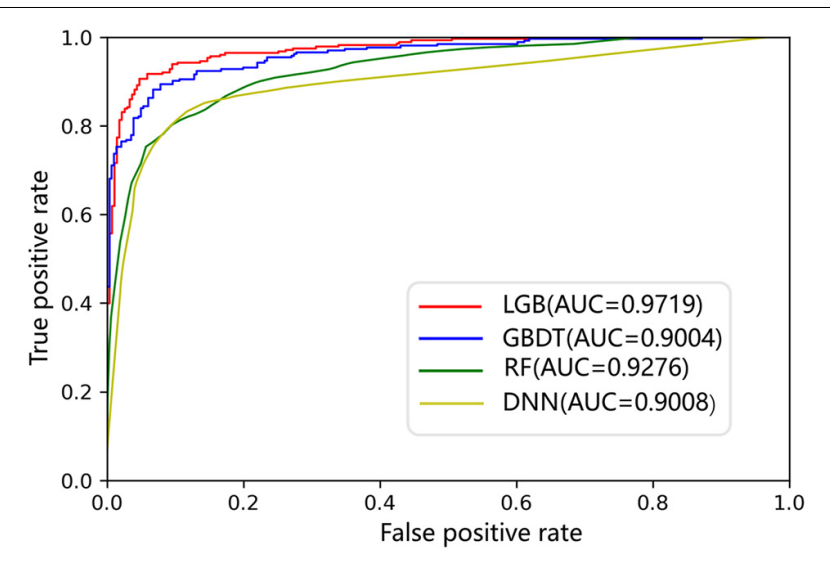

FIGURE 7 | ROCs of different models on the channel-drug interaction dataset.

is finally obtained by averaging every word over the length dimension of the protein. In Figure $\mathbf{2 B}$, every word was encoded in AAindex_word2vec with AAindex indices, which may help generate more efficient vectors of words. For example, when to handle a ion channel sequence through AAindex_word2vec, we divide it into 3-gram and encode every word with AAindex indices, then the "Wordbooks-AAindex" were looked up to obtain a vector for every word. The representation for a protein is finally obtained by averaging every word over the length dimension of the protein.

In the word2vec model, the features learned at each layer are not visual. To explore what information word vectors imply, $t$-distributed stochastic neighbor embedding ( $t$-SNE; van der Maaten and Hinton, 2008), a non-linear dimensionality reduction algorithm, was used to projected the vectors of ion channels from 64-dimensional to 3D space. As shown in Figure 3, the coordinates of points are the values after dimensionality reduction, and the color of points are termed G_X, G_Y, and G_Z, respectively, which are three coefficients derived from the second-order gray model (Xiao et al., 2008). In Figures 3A-C, the coordinates of points are the values after dimensionality reduction, and the color is three coefficients derived from the second-order gray model. In Figures 3D-F, the coordinates of points are the values after dimensionality reduction, and the color is the PI, MW, and extinction coefficient of different segments (ECDF). We can find that word vectors can learn implicitly the three coefficients derived from the second-order gray model (G_X, G_Y, and G_Z), PI, MW, and ECDF.

\section{Representation of Drug}

Due to the complex three-dimensional structure and unique properties of drugs, the characterization of drug performance stored in the computer often lose a lot of information. Fortunately, there are many approaches to represent drugs with different characteristics, which involve molecular diagram, Morgan FPs, SMILES, and so on.

(1) Representing drug with word2vec

The word2vec has been used to generate vectors via SMILES or FP2. There is still a need to know which one is the best choice for this issue. As shown in Figure 4A, the SMILES string can be divided into n-gram. Here, the sequence of the drug was divided into non-overlapping 3-gram, and word2vec algorithm was selected to generate the word vector. In addition, the process to construct FP2_word2vec is shown in Figure 4B.

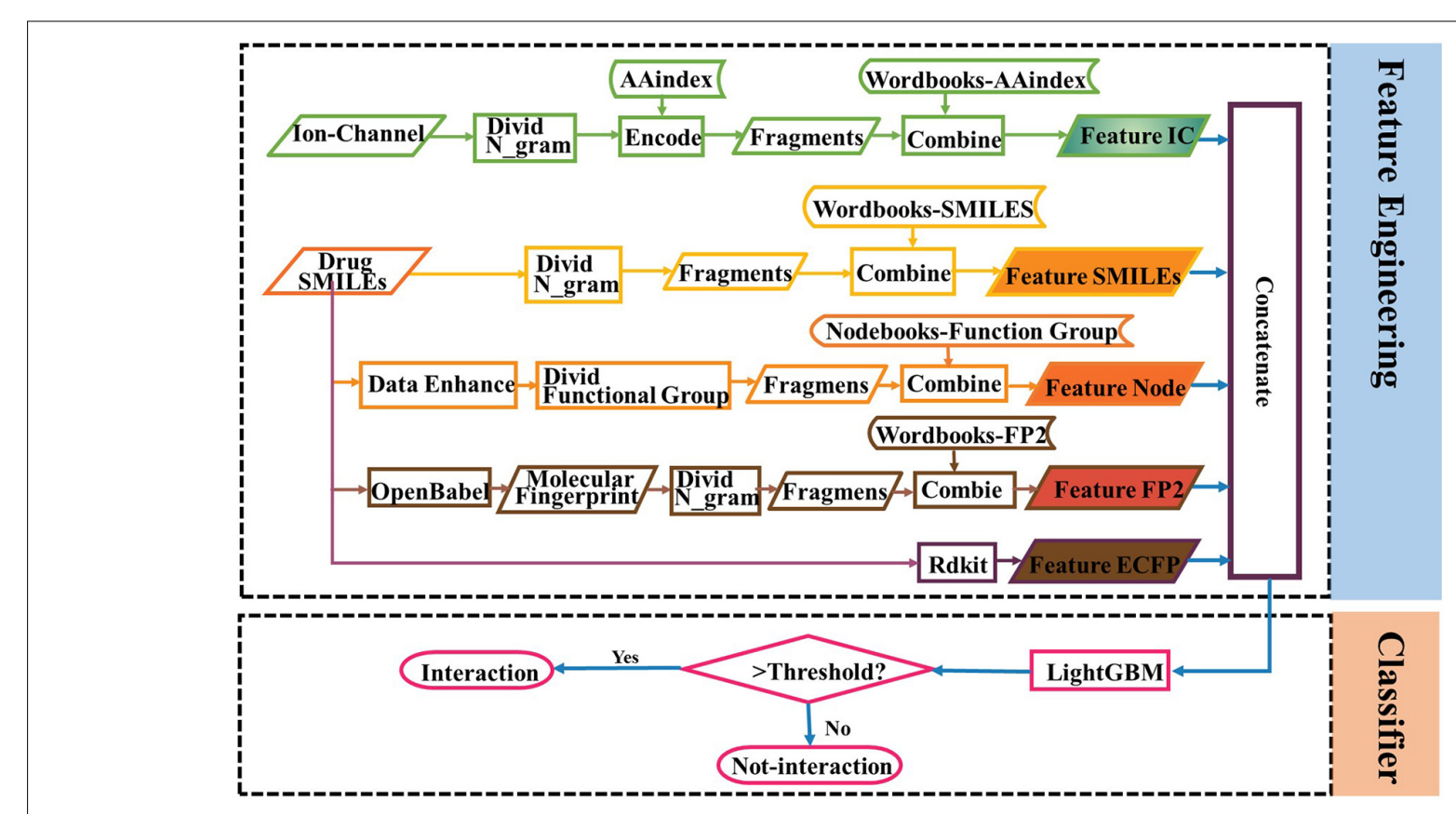

FIGURE 8 | Flowchart of the iCDI-W2vCom model. 
TABLE 5 | Results of the proposed model in channel-drug interaction dataset.

\begin{tabular}{|c|c|c|c|c|c|}
\hline Test & AUC & Acc (\%) & Prec (\%) & Sen (\%) & MCC \\
\hline 1 & 0.9680 & 90.89 & 89.98 & 91.82 & 0.8180 \\
\hline 2 & 0.9650 & 90.53 & 87.50 & 94.07 & 0.8132 \\
\hline 3 & 0.9779 & 92.70 & 92.73 & 93.07 & 0.8579 \\
\hline 4 & 0.9695 & 93.08 & 93.04 & 93.12 & 0.8615 \\
\hline 5 & 0.9711 & 92.53 & 92.65 & 92.42 & 0.8506 \\
\hline Average & $0.9703 \pm 0.0043$ & $91.95 \pm 1.03$ & $91.18 \pm 2.15$ & $92.95 \pm 0.73$ & $0.8402 \pm 0.0205$ \\
\hline
\end{tabular}

(2) Representing drug with node2vec

The node2vec (Grover and Leskovec, 2016) may also represent the drug feature, and it captures the information between nodes in networks (Grohe, 2020; Shen et al., 2021). Here, the node2vec is applied to obtain node features for a complementary characteristic of the drug.

Simplified molecular-input line-entry system strings are divided into functional groups taken as the nodes of the network. The functional group comprises multiple atoms or atomic groups, and its physicochemical properties are fundamental in the pharmacodynamic phase of the mechanisms of action of

TABLE 6 | Performances of different methods on channel-drug interaction dataset.

\begin{tabular}{lccc}
\hline & $\begin{array}{c}\text { RFDT (Wang } \\
\text { et al., 2018) }\end{array}$ & $\begin{array}{c}\text { Wang }(\text { Wang } \\
\text { et al., 2020) }\end{array}$ & The proposed method \\
\hline AUC & $0.8900 \pm 0.0200$ & $0.8895 \pm 0.0146$ & $\mathbf{0 . 9 7 0 3} \pm \mathbf{0 . 0 0 4 3}$ \\
Acc (\%) & $89.10 \pm 1.50$ & $88.82 \pm 0.65$ & $\mathbf{9 1 . 9 5} \pm \mathbf{1 . 0 3}$ \\
Prec (\%) & $87.60 \pm 1.60$ & $88.30 \pm 0.16$ & $\mathbf{9 1 . 1 8} \pm \mathbf{2 . 1 5}$ \\
Sen (\%) & $91.20 \pm 1.90$ & $89.50 \pm 0.73$ & $\mathbf{9 2 . 9 5} \pm \mathbf{0 . 7 3}$ \\
MCC & $0.8060 \pm 0.024$ & $0.7763 \pm 0.0134$ & $\mathbf{0 . 8 4 0 2} \pm \mathbf{0 . 0 2 0 5}$ \\
\hline
\end{tabular}

Bold values mean that they are the best scores compared with other methods.

TABLE 7 | Performance comparisons on Check808.

\begin{tabular}{lcccc}
\hline & RF & DNN & GBDT & LGB \\
\hline A UC & 0.8916 & 0.9058 & 0.9370 & $\mathbf{0 . 9 6 3 0}$ \\
Acc (\%) & 81.68 & 85.64 & 87.25 & $\mathbf{9 0 . 4 7}$ \\
Prec (\%) & 86.23 & 82.22 & 86.16 & $\mathbf{8 9 . 6 4}$ \\
Sen (\%) & 78.10 & 89.44 & 88.43 & $\mathbf{9 1 . 3 5}$ \\
MCC & 0.6388 & 0.7170 & 0.7454 & $\mathbf{0 . 8 0 9 6}$ \\
\hline
\end{tabular}

$R F$, random forest; $D N N$, deep neural network; GBDT, gradient boosting decision tree; LGB, LightGBM. Bold values mean that they are the best scores compared with other methods.

TABLE 8 | Performances of different methods on NR-drug interaction datasets.

\begin{tabular}{lccc}
\hline & $\begin{array}{c}\text { RFDT (Wang } \\
\text { et al., 2018) }\end{array}$ & $\begin{array}{c}\text { Lei }(\text { Wang } \\
\text { et al., 2020) }\end{array}$ & The proposed method \\
\hline AUC & $0.7230 \pm 0.0380$ & $0.8074 \pm 0.0933$ & $\mathbf{0 . 9 0 1 4} \pm \mathbf{0 . 0 3 2 5}$ \\
Acc (\%) & $71.10 \pm 4.60$ & $82.22 \pm 3.17$ & $\mathbf{8 7 . 1 4} \pm \mathbf{3 . 2 3}$ \\
Prec (\%) & $68.00 \pm 12.10$ & $\mathbf{8 4 . 7 4} \pm \mathbf{1 2 . 5 3}$ & $84.73 \pm 4.42$ \\
Sen (\%) & $75.90 \pm 10.00$ & $79.98 \pm 12.70$ & $\mathbf{8 3 . 6 6} \pm \mathbf{4 . 5 5}$ \\
MCC & $0.5790 \pm 0.0400$ & $0.6573 \pm 0.0699$ & $\mathbf{0 . 7 3 3 8} \pm \mathbf{0 . 0 6 7 3}$ \\
\hline
\end{tabular}

Bold values mean that they are the best scores compared with other methods. many drugs (Silva et al., 2019). In the SMILES, the SMILES strings are marked: no mark for single key, " = " for double key, "\#” for triple key, and "(“ or ")" for branch chain". The SMILES of the drug molecules is separated by the special marks, and every part is taken as a node.

The node2vec regards a random path generated by a random walk as a set of words. A data augmentation approach is chosen to generate more paths and get more information between nodes in the networks. As shown in Figure 5, RDKit was used to generate different SMILES strings for the same molecule. These SMILES strings are all valid structures. RDKit generates different SMILES strings by rotating the molecular graph to generate different SMILES strings whose starting atom and the direction of graph enumeration are randomly selected. In the procedure of training node2vec, data augmentation approach can better obtain the connection relation between functional groups and get a better node vector (Tetko et al., 2020).

The process of generating SMILES_node2vec is shown in Figure 6. In Figure $\mathbf{6 A}$, the SMILES strings are divided into function groups, and the Nodebooks-Function Group is generated by the node2vec model. In Figure 6B, SMILES strings were first divided into function groups, and then the "Nodebooks-Function Group" was looked up to obtain a vector for every word. The representation for a drug is finally obtained by averaging every word over the length dimension of the drug.

(3) Representing drug with ECFPs

In the drug database, the drugs in SMILES format (Weininger, 1988) may be further fed into RDKit, to extract their ECFPs (Zhou et al., 2020), such that a drug can be represented by a 1,024-D binary vector.

The multiple ways of representing a molecule and the different levels of uncertainty regarding those representations have been a central part of this expertise. In this paper, we try to improve the accuracy of ion channel-drug interaction by feature combination. In Rayhan et al. (2019), it is shown that an ensemble boosting method performs much better than other methods in DTI prediction. The feature subset of drugs includes SMILES-word2vec (Feature SMILES), FP2word2vec (Feature FP2), SMILES-node2vec (Feature Node), and ECFP (Feature ECFP). As shown in Table 2, the subsets of the feature mentioned above are tested with the LightGBM classifier via fivefold cross-validation, and the feature dimension of the six learners are shown in the last column of the table.

${ }^{4}$ https://www.daylight.com/meetings/summerschool98/course/dave/smiles- 


\section{Prediction Engine}

Once the protein and drug were represented by vectors, some machine learning models would be utilized for the prediction process. We compared the performance of different algorithms involved in LightGBM (LGB; Ke et al., 2017), gradient boosting decision tree (GBDT; Friedman, 2000), random forest (RF; Liaw and Wiener, 2002), and deep neural networks (DNNs) on the ion channel dataset (Pedregosa et al., 2011). All these models were implemented in Python 3 (Python 23.6 ) environment with LightGBM package (Zhang et al., 2017) and Scikit-learn library (Pedregosa et al., 2011).

\section{RESULTS AND DISCUSSION}

The original triplicates workbooks (Orig_word2vec) were used for the first experiment, and five AAindex_word2vec were used for comparison. Results are listed in Table 3. It was found that the AAindex_word2vec for the proteins improved the performance of the classifier greatly. As listed in the tables, bold values mean that they are the best scores compared with other methods.

Comparing the experimental results of original drug expression PF2, the SMILES string as input, and using word2vec to extract features in other articles, this work combined the features of drugs in different descriptions. We can find that the combination of SMILES_word2vec (Feature SMILES), SMILES_node2vec (Feature node), FP2_word2vec (Feature FP2), and ECFP (Feature ECFP) has achieved the optimal effect. Table 4 shows the result comparison of fivefold cross-validation. The descriptors of molecules mentioned above are ambiguous or missing some information, but those descriptors are highly complementary, and experimental findings show that drug feature combination is useful.

The AUC curves of LGB, GBDT, RF, and DNN on the ion channel dataset are shown in Figure 7. The LightGBM approach performs quite high AUCs in the test such that it is selected as the predictor. The parameter values of LightGBM model are num_leaves of 48, max_depth of 9 , learning_rate of 0.03 , n_estimators of 600 , min_child_samples of 3 , and other parameters are set with their default values. The flowchart of the proposed iCDI-W2vCom model is shown in Figure 8. The model inputs the SMILES strings of accessible drugs and the amino acid sequences of ion channels. The feature subsets are fed into the LightGBM predictor for a final prediction with a fivefold cross-validation method.

Predictor was optimized by using AAindex1_word2vec for ion channel and feature combination for drug. Table 5 shows the results of the proposed model on the ion channel-drug interaction dataset via fivefold cross-validation. The ICDIW2vCom based on both the word2vec model and node2vec model has an average AUC of 0.9703 , Acc of $91.95 \%$, precision of $91.18 \%$, sensitivity of $92.95 \%$, MCC of 0.8402 vs. other newly publish methods of $0.8900,89.10 \%, 88.30 \%, 91.20 \%$, 0.8060 , respectively. Thus, our performance has been improved, surpassing other existing classifiers as shown in Table 6.

Using the ion channel-drug interaction benchmark datasets as training dataset and Check808 as an independent test set, different algorithms were tested, and the results are listed in Table 7.

According to the results, the features we generate can very well characterize the channel-drug interactions, and the default classifier LGB has a better generation ability by comparison with RF, DNN, and GBDT.

The proposed method achieved good performance in the NR dataset. The results are listed in Table 8. Compared with the previously published articles (Wang et al., 2018; Wang et al., 2020), the ICDI-W2vCom has great AUC, Acc, Sen, and MCC values such that it gets good robustness.

\section{CONCLUSION}

In the research, the proposed model based on AAindex encoding sequences and word2vec algorithm significantly improved the learning ability of predictors. This inspires us that, in small datasets, coding protein words according to their physical and chemical properties may reduce the number of words in the lexicon, which trained the word2vec model faster and generate a high quality of word. By using conventional protein processing methods and knowledge, the parameters of deep learning could be reduced, and the computation would be simplified. Furthermore, by using the t-SNE algorithm to project the vectors of ion channels from 64-dimensional to 3D space vectors, vectors can learn implicitly features represented by other protein-encoding methods (for example, the gray model) and physicochemical properties. This work suggests that word2vec can also be accepted in ML as many previous works do.

The multiple ways of representing a molecule and the different levels of uncertainty regarding those representations have been a central part of this expertise. Therefore, we try to fuse drug information of different descriptions to represent drugs comprehensively. In this paper, the expression of drugs was enhanced through the combination of different features, and the performance of the classifier was improved greatly.

\section{DATA AVAILABILITY STATEMENT}

The original contributions presented in the study are included in the article/supplementary material, further inquiries can be directed to the corresponding authors.

\section{AUTHOR CONTRIBUTIONS}

$\mathrm{XX}$ conceived and designed the experiments. JZ performed the extraction of features, model construction, model training, and evaluation, and drafted the manuscript. XX and W-RQ supervised the project and revised the manuscript. All authors read and approved the final manuscript.

\section{FUNDING}

This work was supported by the grants from the National Natural Science Foundation of China (Nos. 31860312, 
31760315, and 62062043), Natural Science Foundation of Jiangxi Province, China (Nos. 20202BAB202007 and 20171ACB20023), the Department of Education of Jiangxi

\section{REFERENCES}

Amin, J. B., Leng, X., Gochman, A., Zhou, H. X., and Wollmuth, L. P. (2018). A conserved glycine harboring disease-associated mutations permits NMDA receptor slow deactivation and high $\mathrm{Ca}(2+)$ permeability. Nat. Commun. 9:3748.

Bemis, G., and Kuntz, I. D. (1992). A fast and efficient method for 2D and 3D molecular shape description. J. Comput. Aided Mol. Des. 6, 607-628. doi: $10.1007 /$ bf00126218

Chen, K., Kurgan, L., and Ruan, J. (2006). Prediction of flexible/rigid regions from protein sequences using k-spaced amino acid pairs. BMC Struct. Biol. 7:25. doi: 10.1186/1472-6807-7-25

Chen, R., Liu, X., Jin, S., Lin, J., and Liu, J. (2018). Machine learning for drug-target interaction prediction. Molecules 23:2208. doi: 10.3390/molecules 23092208

Chou, K. C. (2005). Using amphiphilic pseudo amino acid composition to predict enzyme subfamily classes. Bioinformatics 21, 10-19. doi: 10.1093/ bioinformatics/bth466

Clark, A. M., Mcewen, L. R., Gedeck, P., and Bunin, B. (2019). Capturing mixture composition: an open machine-readable format for representing mixed substances. J. Cheminform. 11:33.

Daina, A., and Zoete, V. (2019). Application of the SwissDrugDesign online resources in virtual screening. Int. J. Mol. Sci. 20:4612. doi: 10.3390/ ijms 20184612

Elnaggar, A., Heinzinger, M., Dallago, C., Rehawi, G., Yu, W., Jones, L., et al. (2021). ProtTrans: towards cracking the language of lifes code through selfsupervised deep learning and high performance computing. arXiv [Preprint]. doi: 10.1101/2020.07.12.199554

Friedman, J. (2000). Special invited paper-additive logistic regression: a statistical view of boosting. Ann. Stat. 28, 374-376.

Gabashvili, I. S., Sokolowski, B. H., Morton, C. C., and Giersch, A. B. (2007). Ion channel gene expression in the inner ear. J. Assoc. Res. Otolaryngol. 8, 305-328.

Green, W. N. (1999). Ion channel assembly: creating structures that function. J. Gen. Physiol. 113, 163-170. doi: 10.1085/jgp.113.2.163

Grohe, M. (2020). "word2vec, node2vec, graph2vec, X2vec: towards a Theory of vector embeddings of structured data," in Proceedings of the 39th ACM SIGMOD-SIGACT-SIGAI Symposium on Principles of Database Systems, Portland, OR, 1-19. doi: 10.1145/3375395.3387641

Grover, A., and Leskovec, J. (2016). "node2vec: scalable feature learning for networks," in Proceedings of the 22nd ACM SIGKDD International Conference on Knowledge Discovery and Data Mining, San Francisco, CA. doi: 10.1145/ 2939672.2939754

Huang, K., Fu, T., Glass, L. M., Zitnik, M., Xiao, C., and Sun, J. (2020). DeepPurpose: a deep learning library for drug-target interaction prediction. Bioinformatics 36, 5545-5547. doi: 10.1093/bioinformatics/btaa1005

Jaeger, S., Fulle, S., and Turk, S. (2017). Mol2vec: unsupervised machine learning approach with chemical intuition. J. Chem. Inf. Model. 58, 27-35. doi: 10.1021/ acs.jcim.7b00616

Jaeger, S., Fulle, S., and Turk, S. (2018). Mol2vec: unsupervised machine learning approach with chemical intuition. J. Chem. Inf. Model. 58, 27-35. doi: 10.1021/ acs.jcim.7b00616

Jiao, Y., and Du, P. (2016). Performance measures in evaluating machine learning based bioinformatics predictors for classifications. Quant. Biol. 4, 320-330. doi: 10.1007/s40484-016-0081-2

Karimi, M., Wu, D., Wang, Z., and Shen, Y. (2019). DeepAffinity: interpretable deep learning of compound-protein affinity through unified recurrent and convolutional neural networks. Bioinformatics 35, 3329-3338. doi: 10.1093/ bioinformatics/btz111

Kaufmann, D., Tietze, A. A., and Tietze, D. (2019). In silico analysis of the subtype selective blockage of KCNA ion channels through the mu-conotoxins PIIIA, SIIIA, and GIIIA. Mar. Drugs 17:180. doi: 10.3390/md17030180

Ke, G., Meng, Q., Finley, T., Wang, T., Chen, W., Ma, W., et al. (2017). "LightGBM: a highly efficient gradient boosting decision tree," in The 31st International
Province (Nos. GJJ160866 and GJJ180703), and the International Cooperation Project of the Ministry of Science and Technology, China (No. 2018-3-3).

Conference on Neural Information Processing Systems (NIPS 2017), Long Beach, CA, 3149-3157.

Knowles, J., and Gromo, G. (2003). Target selection in drug discovery. Nat. Rev. Drug Discov. 2, 63-69. doi: 10.1038/nrd986

Liaw, A., and Wiener, M. (2002). Classification and regression by randomForest. $R$ News 2, 18-22.

O’Boyle, N. M. (2012). Towards a Universal SMILES representation - A standard method to generate canonical SMILES based on the InChI. J. Cheminformatics $4: 22$.

Pedregosa, F., Varoquaux, G., Gramfort, A., Michel, V., Thirion, B., Grisel, O., et al. (2011). Scikit-learn: machine learning in python. J. Mach. Learn. Res. 12, 2825-2830.

Qiang, J., Kai, L., Lu, W. J., Shuang, L., Chen, X., Liu, X. J., et al. (2018). Identification of small-molecule ion channel modulators in C. elegans channelopathy models. Nat. Commun. 9:3941.

Qiu, W., Lv, Z., Hong, Y., Jia, J., and Xiao, X. (2020). BOW-GBDT: a GBDT classifier combining with artificial neural network for identifying GPCR drug interaction based on wordbook learning from sequences. Front. Cell Dev. Biol. 8:623858. doi: 10.3389/fcell.2020.623858

Rayhan, F., Ahmed, S., Md Farid, D., Dehzangi, A., and Shatabda, S. (2019). CFSBoost: cumulative feature subspace boosting for drug-target interaction prediction. J. Theor. Biol. 464, 1-8. doi: 10.1016/j.jtbi.2018.12.024

Reczko, M., and Bohr, H. (1994). The DEF data-base of sequence based protein fold class predictions. Nucleic Acids Res. 22, 3616-3619.

Roepke, T. K., King, E. C., Reyna-Neyra, A., Paroder, M., Purtell, K., Koba, W., et al. (2009). Kcne2 deletion uncovers its crucial role in thyroid hormone biosynthesis. Nat. Med. 15, 1186-1194. doi: 10.1038/nm. 2029

Shen, Z., Deng, S.-P., and Huang, D.-S. (2020). RNA-protein binding sites prediction via multi scale convolutional gated recurrent unit networks. Bioinformatics 17, 1741-1750. doi: 10.1109/tcbb.2019.291 0513

Shen, Z.-A., Luo, T., Zhou, Y.-K., Yu, H., and Du, P.-F. (2021). Predicting ncRNAprotein interactions with deep graph neural networks. Brief. Bioinformatics 17:bbab051.

Silva, J., Brigido, H., Albuquerque, K., Carvalho, J. M., and Dolabela, M. (2019). Flavopereirine-An alkaloid derived from Geissospermum vellosiiPresents leishmanicidal activity in vitro. Molecules 24:785. doi: 10.3390/ molecules 24040785

Sumino, A., Sumikama, T., Uchihashi, T., and Oiki, S. (2019). High-speed AFM reveals accelerated binding of agitoxin- 2 to a $\mathrm{K}(+)$ channel by induced fit. Sci. Adv. 5:eaax0495. doi: 10.1126/sciadv.aax0495

Tetko, I., Karpov, P., Deursen, R. V., and Godin, G. (2020). State-of-the-art augmented NLP transformer models for direct and single-step retrosynthesis. Nat. Commun. 11:5575.

Tinaquero, D., Crespo-Garcia, T., Utrilla, R. G., Nieto-Marin, P., GonzalezGuerra, A., Rubio-Alarcon, M., et al. (2020). The p.P888L SAP97 polymorphism increases the transient outward current (Ito,f) and abbreviates the action potential duration and the QT interval. Sci. Rep. 10:10707.

Tsubaki, M., Tomii, K., and Sese, J. (2019). Compound-protein interaction prediction with end-to-end learning of neural networks for graphs and sequences. Bioinformatics 35, 309-318. doi: 10.1093/bioinformatics/b ty 535

van der Maaten, L., and Hinton, G. (2008). Visualizing data using t-SNE. J. Mach. Learn. Res. 9, 2579-2605.

Wang, D., Liang, Y., and Xu, D. J. B. (2019). Capsule network for protein posttranslational modification site prediction. Bioinformatics 35, 2386-2394. doi: 10.1093/bioinformatics/bty977

Wang, L., You, Z. H., Chen, X., Yan, X., Liu, G., and Zhang, W. (2018). RFDT: a rotation forest-based predictor for predicting drug-target interactions using drug structure and protein sequence information. Curr. Protein Pept. Sci. 19, 445-454. doi: 10.2174/1389203718666161114111656 
Wang, L., You, Z. H., Li, L. P., Yan, X., Zhang, W., Song, K. J., et al. (2020). Identification of potential drug-targets by combining evolutionary information extracted from frequency profiles and molecular topological structures. Chem. Biol. Drug Des. 96, 758-767. doi: 10.1111/cbdd.13599

Wang, P., Huang, X., Qiu, W., and Xiao, X. (2020). Identifying GPCR-drug interaction based on wordbook learning from sequences. BMC Bioinformatics 21:150. doi: 10.1186/s12859-020-3488-8

Weininger, D. (1988). smiles, a chemical language and information-system .1. Introduction to methodology and encoding rules. J. Chem. Inf. Comput. Sci. 28, 31-36. doi: 10.1021/ci00057a005

Xiao, X., Lin, W.-Z., and Chou, K.-C. (2008). Using grey dynamic modeling and pseudo amino acid composition to predict protein structural classes. J. Comput. Chem. 29, 2018-2024. doi: 10.1002/jcc.20955

Xiao, X., Min, J.-L., Wang, P., and Chou, K.-C. (2013). iCDI-PseFpt: identify the channel-drug interaction in cellular networking with PseAAC and molecular fingerprints. J. Theor. Biol. 337, 71-79. doi: 10.1016/j.jtbi.2013. 08.013

Yamanishi, Y., Araki, M., Gutteridge, A., Honda, W., and Kanehisa, M. (2008). Prediction of drug-target interaction networks from the integration of chemical and genomic spaces. Bioinformatics 24, i232-i240.

Yang, K. K., Wu, Z., Bedbrook, C. N., and Arnold, F. H. (2018). Learned protein embeddings for machine learning. Bioinformatics 34, 2642-2648. doi: 10.1093/ bioinformatics/bty 178

Zhang, H., Si, S., and Hsieh, C.-J. (2017). GPU-acceleration for large-scale tree boosting. arXiv [Preprint]. Avaliable online at: https://arxiv.org/abs/1706.08359 (accessed June 26, 2017).
Zhang, Y. F., Wang, X., Kaushik, A. C., Chu, Y., Shan, X., Zhao, M. Z., et al. (2019). SPVec: a Word2vec-inspired feature representation method for drugtarget interaction prediction. Front. Chem. 7:895. doi: 10.3389/fchem.2019.0 0895

Zhou, J. P., Chen, L., Wang, T., and Liu, M. (2020). iATC-FRAKEL: a simple multi-label web server for recognizing anatomical therapeutic chemical classes of drugs with their fingerprints only. Bioinformatics 36, 3568-3569. doi: 10. 1093/bioinformatics/btaa166

Conflict of Interest: The authors declare that the research was conducted in the absence of any commercial or financial relationships that could be construed as a potential conflict of interest.

Publisher's Note: All claims expressed in this article are solely those of the authors and do not necessarily represent those of their affiliated organizations, or those of the publisher, the editors and the reviewers. Any product that may be evaluated in this article, or claim that may be made by its manufacturer, is not guaranteed or endorsed by the publisher.

Copyright (c) 2021 Zheng, Xiao and Qiu. This is an open-access article distributed under the terms of the Creative Commons Attribution License (CC BY). The use, distribution or reproduction in other forums is permitted, provided the original author(s) and the copyright owner(s) are credited and that the original publication in this journal is cited, in accordance with accepted academic practice. No use, distribution or reproduction is permitted which does not comply with these terms. 